Activin-like kinase receptors 17

Adenovirus 95

Alkaline phosphatase 109

Amelogenesis imperfecta 7

Amelogenin 7

Apocrine glands 187

Arterial abnormalities, fetus 195

Biomineralization 7

Bone morphogenetic protein(s) 28, 109

Brachial plexus, cat 205

Cat brachial plexus 205

C2C12 cells 28

Cell fusion 67

Chondrocyte 41

Chondrogenesis 79

Chondroinduction 41

Colla1-GFP 120

Collagen tube 153

Defensin 187

Degradation rate, collagen chambers 153

Demineralized bone powder 41

Dog skeletal muscles 178

Dura mater 54

Extracellular matrix 87

Fetus, vertebral artery duplications 195

Fibre types, dog skeletal muscles 178

Fibroblast growth factors 54

Fracture healing 95

Gene expression 41

Green fluorescent protein 120

Histochemistry, fibre metabolic profile 178 Hypoxia-inducible factor- $1 \alpha 87$

IgA 187

Interleukin-4, cell fusion 67

Keloids 87

Keratin 2e 169

Keratinocyte 134

\section{Lysozyme 187}

Macrophage, cell fusion 67

Mammary gland development 169

Marrow stromal cells 109

Mesenchymal stem cells 109

Microarray, gene expression 28

Mucin 187

Myosin heavy chain 178

Myotube 67

\section{NFATC2 67}

Nipple 169

Odontogenesis 7, 120

Oral mucosa, tissue-engineered 134

Osteoblast 95

- differentiation 28

Osteogenesis 109, 120

Parathyroid-hormone-related protein 169

Peripheral nerve 153

Perlecan 79

Progenitor cells 120

Prostaglandin $F_{2 \alpha} 67$

Regeneration, peripheral nerves 153

Replacement teeth 17

Representational difference analysis, chondroinduction 41

Runx2/Cbfa1 28

Skeletal muscles, dog 178

Stem cells 120

Sutures 54

TGF- $\beta$ family member signaling 17

Tissue engineering 134

- fibrosis 87

- interactions 54

Transcription factors 109

Transgenics 7

Vascular endothelial growth factor 87

Vertebrobasilar system 195

\section{KARGER}

(C) 2004 S. Karger AG, Basel

Fax + 41613061234

E-Mail karger@karger.ch

www.karger.com
Accessible online at: www. karger.com/cto 